\title{
Fractal Butterflies of Chiral Fermions in Bilayer Graphene: Phase Transitions and Emergent Properties
}

\author{
Areg Ghazaryan and Tapash Chakraborty \\ Department of Physics and Astronomy, University of Manitoba, Winnipeg, Canada R3T 2N2
}

(Dated: October 30, 2018)

\begin{abstract}
We report on our studies of fractal butterflies in biased bilayer graphene in the fractional quantum Hall effect (FQHE) regime. We have considered the case when the external periodic potential is present in one layer and have illustrated the effect of varying both the periodic potential strength and the bias voltage on the FQHE and the butterfly energy gaps. Interestingly, the butterfly spectra exhibits remarkable phase transitions between the FQHE gap and the butterfly gap for chiral electrons in bilayer graphene, by varying either the periodic potential strength or the bias voltage. We also find that, in addition to those phase transitions, by varying the bias voltage one can essentially control the periodic potential strength experienced by the electrons.
\end{abstract}

Graphene 2, 3] placed on a hexagonal substrate with a twist is expected to display the Hofstadter butterfly pattern [4, 5] in the energy spectrum of non-interacting Dirac fermions, when subjected to a perpendicular magnetic field. The unique butterfly pattern was indeed discovered in 2013 in monolayer and bilayer graphene [68], placed on a hexagonal boron nitride substrate with rotational misalignment between graphene and the substrate. That arrangement resulted in the Moiré pattern which actually introduces a large-scale periodicity in the Hamiltonian of the system, and the fractal butterfly pattern was the result of splitting of the Moiré minibands (secondary Dirac cones) by the magnetic field that are exhibited in the magnetoconductance probe 9]. Interestingly, a mathematical proof of the presence of the fractal pattern in the butterfly spectrum (the 'Ten Martini Problem') is also available in the literature [10]. After that exciting experimental discovery of the fractal butterflies, more recent studies (both theoretical [11] and experimental 12]) have focused on the influence of the electron-electron interaction on the butterfly spectrum. Electronic properties of Dirac fermions in monolayer and bilayer graphene have been exhaustively studied in recent years 2, 3, 13]. In a strong perpendicular magnetic field, interacting Dirac fermions [14] display the fractional quantum Hall Effect (FQHE) states [15] in monolayer [16] and bilayer graphene [13, 17], that has also been experimentally observed [18]. The interaction effects in the fractal butterflies are, of course, more complex in the fractional quantum Hall effect regime, where one observes an interplay between the quantum Hall effect gap and the Hofstadter gap [19]. Interestingly, in this work we find that the butterfly spectra exhibit remarkable phase transitions for chiral electrons in bilayer graphene, where in addition to the phase transitions between the Hofstadter gap and the FQHE gap, one can essentially control the periodic potential strength experienced by the electrons by varying the bias voltage.

We consider bilayer graphene with Bernal (AB) stacking in an external periodic potential with square symmetry [1, 20 22]. We label the layers of bilayer graphene by the indices 1,2 and assume that the periodic potential is present only in layer 1 . Considering that the layers are stacked such that the $B_{1}$ and the $A_{2}$ sites are vertically aligned, the single-particle Hamiltonian of this system in a magnetic field (without the periodic potential) is written $[2,3,13,23]$

$$
\mathcal{H}_{\xi}^{b i}=\xi\left(\begin{array}{cccc}
\frac{U}{2} & v_{F^{\prime}} \pi_{-} & 0 & 0 \\
v_{F} \pi_{+} & \frac{U}{2} & \xi \gamma_{1} & 0 \\
0 & \xi \gamma_{1} & -\frac{U}{2} & v_{F} \pi_{-} \\
0 & 0 & v_{F} \pi_{+} & -\frac{U}{2}
\end{array}\right),
$$

where $\pi_{ \pm}=\pi_{x} \pm i \pi_{y}, \boldsymbol{\pi}=\mathbf{p}+e \mathbf{A} / c, \mathbf{p}$ is the two-dimensional electron momentum, $\mathbf{A}=(0, B x, 0)$ is the vector potential, $v_{F} \approx 10^{6} \mathrm{~m} / \mathrm{s}$ is the Fermi velocity in graphene, $U$ is the inter-layer bias voltage, $\gamma_{1} \approx 0.4 \mathrm{eV}$ is the interlayer hopping integral and $\xi=1$ for $K$ valley and $\xi=-1$ for $K^{\prime}$ valley. The corresponding wave function is described by a fourcomponent spinor $\left(\psi_{A_{1}}, \psi_{B_{1}}, \psi_{A_{2}}, \psi_{B_{2}}\right)^{T}$ for valley $K$ and $\left(\psi_{B_{2}}, \psi_{A_{2}}, \psi_{B_{1}}, \psi_{A_{1}}\right)^{T}$ for valley $K^{\prime}$, where $\psi_{A}$ and $\psi_{B}$ are wave functions of sublattices $\mathrm{A}$ and $\mathrm{B}$, respectively. We consider the fully spin-polarized electron system and therefore disregard the Zeeman energy. The eigenfunction of the Hamiltonian (1) then has the form

$$
\Psi_{n, j}=\left(\begin{array}{c}
\xi C_{1} \varphi_{n-1, j} \\
C_{2} \varphi_{n, j} \\
C_{3} \varphi_{n, j} \\
\xi C_{4} \varphi_{n+1, j}
\end{array}\right),
$$

where $C_{1}, C_{2}, C_{3}, C_{4}$ are constants and $\varphi_{n, j}$ is the electron wave function in the $n$-th Landau level (LL) with the parabolic dispersion, taking into account the periodic boundary conditions (PBC) [15, 24]. In the wave function (2) the LL index $n$ can take the values $-1,0,1, \ldots$ and we assume that if the LL index of $\varphi_{n, j}$ is negative then it is identically equal to zero. In this case, for $n=-1$ the wave function (21) is $\Psi_{-1, j}=\left(0,0,0, \varphi_{0, j}\right)$ and there is only one energy level corresponding to this case. For $n=0, C_{1}=0$ and there are three energy states in this case. Following the convention for the indexing the energy levels introduced in 13], we label the states for $n=-1$ and $n=0$ as $0_{i}^{(\xi)}$, where $i=-2,-1,1,2$ is 


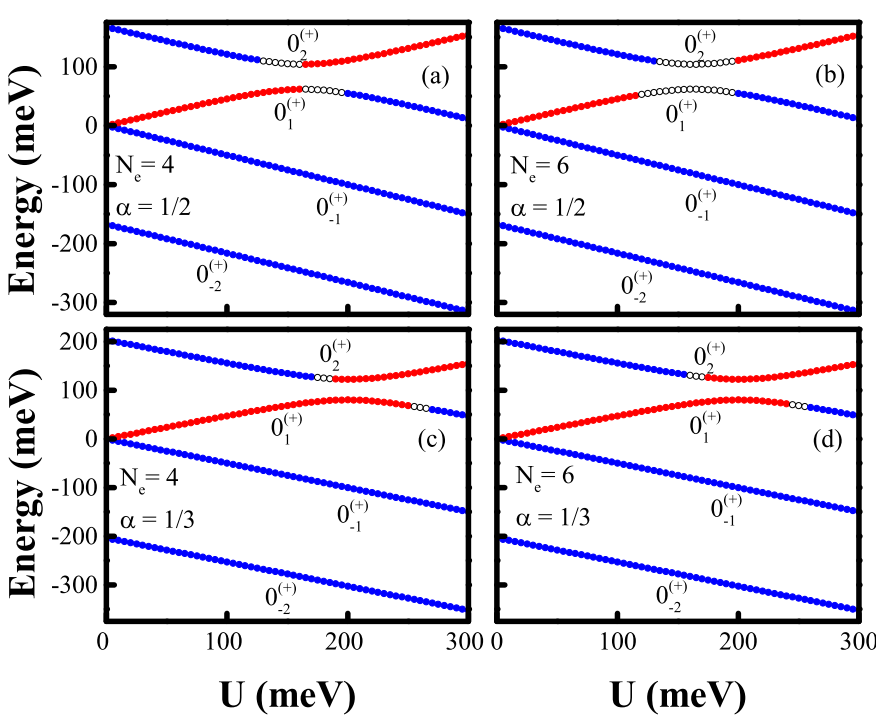

FIG. 1: The Landau levels for $n=-1$ and $n=0$ as a function of the bias voltage $\mathrm{U}$ for two values of $\alpha$, with periodic potential strength $V_{0}=20 \mathrm{meV}$. The numbers next to the curves denote the corresponding Landau level as described in the text. The regions where the gap corresponds to the FQHE (butterfly) gap are drawn as blue (red) dots. (a) and (c) correspond to the system with $N_{e}=4$ and (b) and (d) to $N_{e}=6$.

the label of states in ascending order of the energy values. In particular, for valley $K$ the state corresponding to $n=-1$ has the index $0_{-1}^{(+)}$and $0_{1}^{(-)}$for valley $K^{\prime}$.

The complete many-body Hamiltonian for this system can be written as

$$
\mathcal{H}=\sum_{i}^{N_{e}}\left[\mathcal{H}_{\xi}^{b i}+V\left(x_{i}, y_{i}\right)\right]+\frac{1}{2} \sum_{i \neq j}^{N_{e}} V_{i j}
$$

where the second term is the periodic potential, which is nonzero only for the components of layer 1 and the last term is the Coulomb interaction. We disregard the valley mixing terms (short-range interaction energies) due to the periodic potential and the Coulomb interaction $[19,25]$. Both the inter-layer bias voltage and the periodic potential break spatial inversion symmetry and therefore the valley degeneracy is lifted in this system [26]. For the many-body problem we consider a system of finite number $N_{e}$ of electrons in a toroidal geometry, i.e., the size of the system is $L_{x}=M_{x} a_{0}$ and $L_{y}=M_{y} a_{0}$ ( $M_{x}$ and $M_{y}$ are integers, $a_{0}$ is the period of the external potential) and apply periodic boundary conditions (PBC) in order to eliminate the boundary effects. Defining the parameter $\alpha=\phi_{0} / \phi$ (the inverse of the magnetic flux through the unit cell measured in units of the flux quantum), where $\phi=B a_{0}^{2}$ is the magnetic flux through the unit cell of the periodic potential and $\phi_{0}=h c / e$ the flux quantum, we have $N_{s} /\left(M_{x} M_{y}\right)=1 / \alpha$, where $N_{s}$ is the number of magnetic flux quanta passing through the system or, alternatively, it describes the LL degeneracy for each value of the spin and valley index and

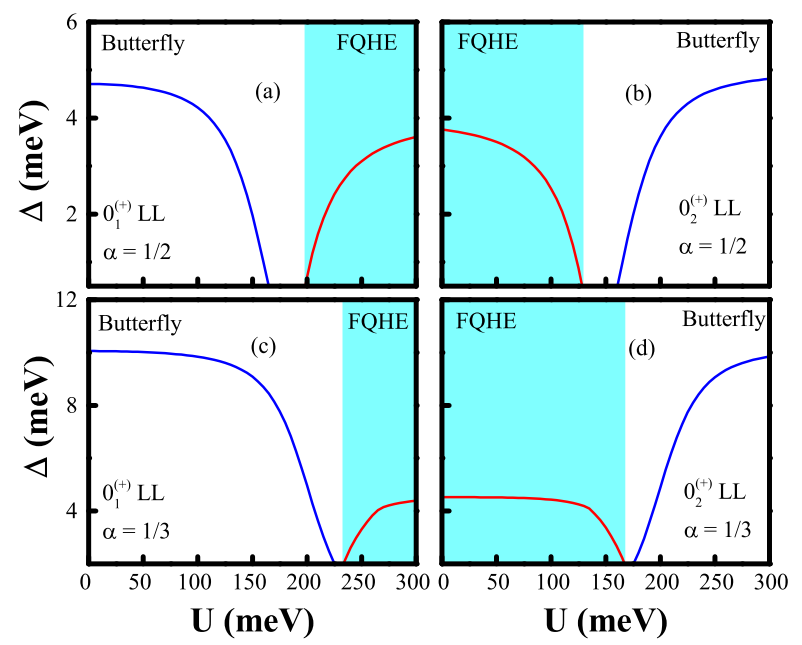

FIG. 2: The dependence of both the FQHE and the butterfly gaps for $N_{e}=4$ electrons on the bias voltage $U$ and the Landau levels $0_{1}^{(+)}$and $0_{2}^{(+)}$for two values of $\alpha$, with the periodic potential strength $V_{0}=20 \mathrm{meV}$. The line corresponding to the FQHE (butterfly) case is depicted in red (blue). The region of the bias voltage $U$ where the gap corresponds to the FQHE gap is marked in cyan.

takes integer values. The filling factor is defined as $\nu=p / q=N_{e} / N_{s}$, where $p$ and $q$ are again coprime integers. In order to solve this problem we first construct the Hamiltonian matrix using the Hamiltonian operator (3) and the many-body states $\left|j_{1}, j_{2}, \ldots, j_{N_{e}}\right\rangle$ (besides $j_{i}$, each single-particle state is characterized by the LL and valley indices which are not shown, but are implicitly assumed to be included in the indices $j_{i}$ ) which are constructed from the single-particle eigenvectors (2). After that we use the center of mass $(\mathrm{CM})$ translation algebra 15, 19, 25, 27, 28] and the eigenstates of CM translations to bring the Hamiltonian matrix into block diagonal form, where each block can then be diagonalized using the exact diagonalization procedure [15].

In this work we consider the filling factor $\nu=1 / 3$ for a fully spin polarized system and for $\alpha=1 / 2$ and $\alpha=1 / 3$. The period of the external potential is taken to be $a_{0}=20 \mathrm{~nm}$, and the inter layer hopping integral to be $\gamma_{1}=30 \mathrm{meV}$ [17], which can be achieved by applying an in-plane magnetic field [13]. In Fig. [1 the dependence of the single-particle LL $0_{i}^{(+)}$on the bias-voltage is presented for the $K$ valley. The color of the filled dots indicate the regions of the bias voltage where for $N_{e}=4$ (a,c) and $N_{e}=6(\mathrm{~b}, \mathrm{~d})$ the gap corresponds to the butterfly region or the FQHE region. The periodic potential strength $V_{0}$ is taken to be $V_{0}=20 \mathrm{meV}$. In order to understand the phase transitions observed in Fig. 1 the wave functions and also the impact of the bias voltage on these wave functions should be analyzed for each LL. As mentioned above, for level $0_{-1}^{(+)}$the wave function has a non-zero component only in layer 2 and this remains true for all values of the bias voltage. Due to the fact 
that the periodic potential is present only in the first layer, the wave functions and therefore also the FQHE gaps do not depend on the bias voltage for LL $0_{-1}^{(+)}$and there is no phase transition in this case. In the LL $0_{-2}^{(+)}$, for $U=0$ the electrons are mostly located in the layer 2 , although they have small probability of being in layer 1. Increasing the bias voltage, both the single-particle and the many-particle system become even more polarized in layer 2 , and therefore the periodic potential has a negligible impact on this level as well and we observe the FQHE gap for all values of $U$. The situation is different for LL $0_{1}^{(+)}$and $0_{2}^{(+)}$. For $U=0$ the electrons in $0_{1}^{(+)}$ are mostly localized in layer 1 and therefore the periodic potential has a drastic impact in this case.

In monolayer graphene the magnitude of the periodic potential $V_{0}=20 \mathrm{meV}$ for $N_{e}=4$ and $N_{e}=6$ and for both values of $\alpha$, closeis the FQHE gap and opens the butterfly gap [19]. It should be pointed out that for $\alpha=1 / 2$, there is no gap in the butterfly spectrum for the non-interacting electrons, but the electron-electron interaction opens a gap [11. However, for $\alpha=1 / 3$ the gap is due to both the Hofstadter gap observed in the single-particle case and due to the contribution from the electron-electron interaction. Therefore, the gap for LL $0_{1}^{(+)}$correspond to the butterfly gap for low values of the bias voltage $U$. The electrons in $0_{2}^{(+)}$are mostly localized in layer 2 and therefore the periodic potential has only a minor effect on them. Hence for the $0_{2}^{(+)}$ LL, the FQHE gap is observed for low values of the bias voltage $U$. By increasing the bias voltage $U$ there is an anticrossing between these two LLs $\left(0_{1}^{(+)}\right.$and $\left.0_{2}^{(+)}\right)$ and thereafter the layer polarization in each LL changes drastically. In particular, for the LL $0_{1}^{(+)}$at the bias voltage $U=250 \mathrm{meV}$ the probability of electrons being localized in layer 2 and for the electrons in LL $0_{2}^{(+)}$to be localized in layer 1 are already $\approx 0.95$. This results in a phase transition in both LLs, namely the gap in the LL $0_{1}^{(+)}$which initially represented the butterfly gap now correspond to the FQHE gap. The opposite behavior occurs for the LL $0_{2}^{(+)}$.

The closure of the FQHE gap by the external periodic potential also occurs in monolayer graphene [19]. However, in bilayer graphene one can control the actual strength of the periodic potential experienced by the electrons essentially by applying the bias voltage. The implications of this interesting result will be discussed below. The described behavior is almost the same for both values of $\alpha=1 / 2$ and $\alpha=1 / 3$. The essential difference between these two cases is that the butterfly gap for $\alpha=1 / 3$ is substantially bigger than that for $\alpha=1 / 2$, and therefore the value of the bias voltage $U$, which is needed to observe the phase transition from the butterfly region to the FQHE region for the LL $0_{1}^{(+)}$is bigger than that for $\alpha=1 / 2$. We have done similar studies for the $K^{\prime}$ valley and similar phase transitions were observed in that case as well for the LLs $0_{-2}^{(-)}$and $0_{-1}^{(-)}$in the negative energy

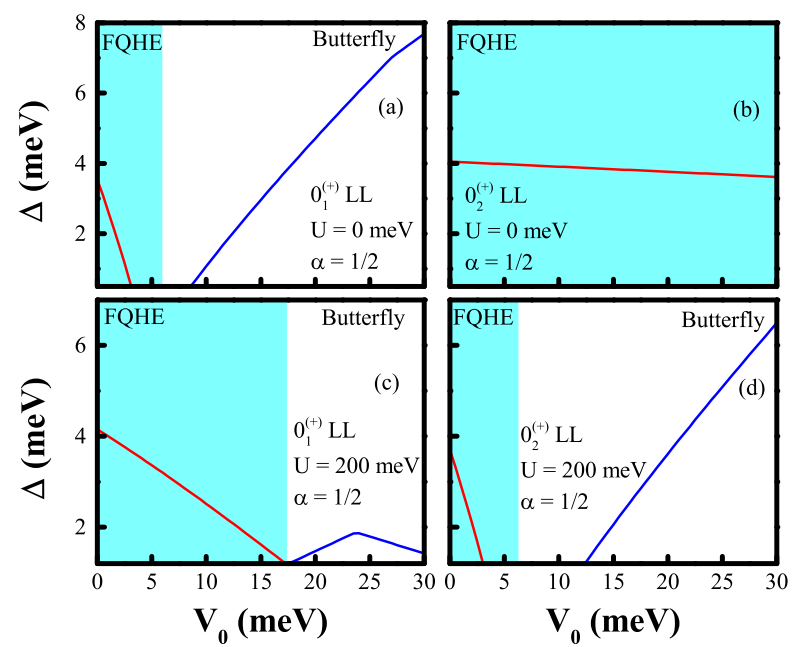

FIG. 3: The dependence of both the FQHE and the butterfly gaps for $N_{e}=4$ electrons on the periodic potential strength $V_{0}$ for Landau levels $0_{1}^{(+)}$and $0_{2}^{(+)}$and for the bias voltages $U=0$ $\mathrm{meV}$ and $U=200 \mathrm{meV}$, and $\alpha=1 / 2$. The line corresponding to the FQHE (butterfly) is depicted in red (blue) color. The region of the periodic potential strength $V_{0}$ where the gap corresponds to FQHE gap is marked in cyan.

region.

In Fig. 2, the dependence of both the FQHE and the butterfly gaps for $N_{e}=4$ electrons on the bias voltage $U$ for Landau levels $0_{1}^{(+)}$and $0_{2}^{(+)}$and for two values of $\alpha$ is shown for $V_{0}=20 \mathrm{meV}$. The region of the bias voltage $U$ where the gap corresponds to the FQHE and the butterfly gap is also indicated. As was already pointed out, the layer polarization of the electrons changes drastically near the anticrossing point of the LLs $0_{1}^{(+)}$and $0_{2}^{(+)}$and the consequence of that can be clearly seen in the dependence of the gaps on the bias voltage $U$. In Fig. 2, in the regions further away from the anticrossing point the gaps are almost constant and fall rapidly to zero when approaching the anticrossing point. Also it can be clearly seen that the FQHE gap is almost the same for both LLs and for both values of $\alpha$, whereas the butterfly gap is almost twice as big for $\alpha=1 / 3$ compared to that of $\alpha=1 / 2$ as explained above.

The dependence of both the FQHE and the butterfly gaps for $N_{e}=4$ electrons on the periodic potential strength $V_{0}$ for Landau levels $0_{1}^{(+)}$and $0_{2}^{(+)}$is shown in Fig. 3f for the bias voltages $U=0 \mathrm{meV}$ and $U=200 \mathrm{meV}$, and for $\alpha=1 / 2$. The same dependence for $\alpha=1 / 3$ is shown in Fig. (4) The region of the periodic potential strength $V_{0}$ where the gap corresponds to the FQHE and the butterfly gaps is also indicated. In Fig. 3 and 4. similar phase transitions between the FQHE and the butterfly gaps is observed as well, although the dependence of the gap on the periodic potential strength $V_{0}$ is almost linear in comparison to the dependence on the bias voltage $U$. No phase transition is observed for the 


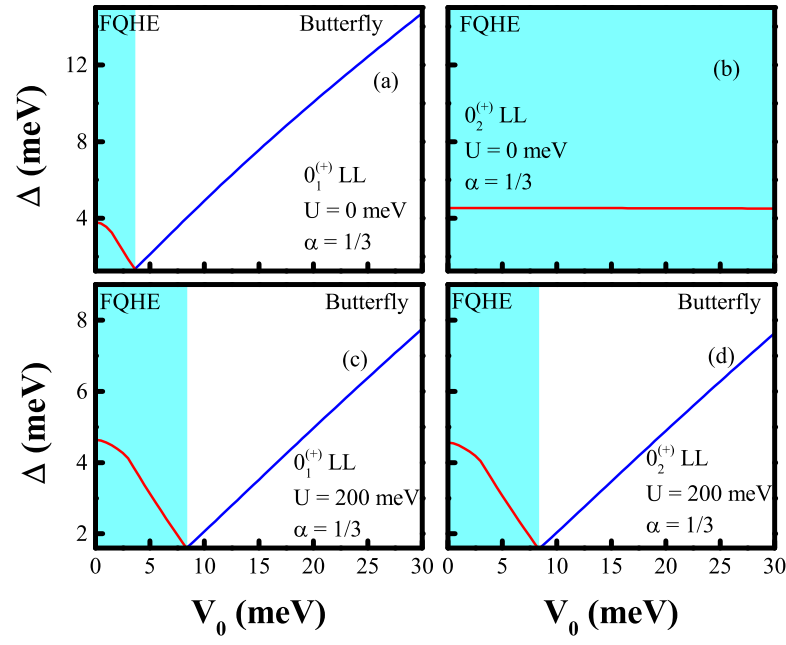

FIG. 4: Same as in Fig. [3 but for $\alpha=1 / 3$.

LL $0_{2}^{(+)}$and $U=0 \mathrm{meV}$, because as was noted above, in this case the electrons are mostly localized in layer 2 and the periodic potential has almost no impact on the physical system. This feature is not observed for the case of $U=200 \mathrm{meV}$, because as we noted above, application of a bias voltage gradually polarizes the electrons for the LL $0_{2}^{(+)}$from layer 2 to layer 1 and the effect of the periodic potential is apparent already for $U=200 \mathrm{meV}$. A similar behavior is observed for $\operatorname{LL} 0_{1}^{(+)}$, where the application of the bias voltage results in widening of the FQHE gap region for both cases of $\alpha$. While the butterfly gap is almost linear for $\alpha=1 / 3$ in Fig. 4 , which indicates that the main contribution here is due to the Hofstadter gap (single particle), for $\alpha=1 / 2$ (Fig. 3) it deviates from the linear behavior in some cases. This indicates that the butterfly gap due to the electron-electron interaction is highly non-trivial.

While the closure of the FQHE gap and opening of the butterfly gap due to the external periodic potential occurs in monolayer graphene [19], one cannot control the periodic potential strength in that system. Therefore, there is no direct method to vary the periodic potential strength in the experiment. In bilayer graphene, as our present work indicates, this can be achieved by applying the bias voltage on the sample. Based on the observations above, variation of the bias voltage offers us the ability to control the polarization of the electrons between the two layers, which essentially translates to the control of the strength of the periodic potential, and at the same time exhibits the phase transition between the FQHE gap and the butterfly gap. This can have significant implications for experimental realization of the fractal butterflies in the FQHE regime.

In conclusion, we have utilized the exact diagonalization scheme to study the FQHE and the butterfly gaps in bilayer graphene under the applied interlayer bias voltage and for the filling factor $\nu=1 / 3$. We have considered the case when the external periodic potential is present in one layer and have illustrated the effect of varying both the periodic potential strength and the bias voltage on the FQHE and the butterfly gaps. Two values of the parameter $\alpha$ were considered, namely $\alpha=1 / 2$ and $\alpha=1 / 3$. We found that by varying either the periodic potential strength or the bias voltage for some Landau levels in both valleys, a phase transition from the FQHE gap to the butterfly gap or vice versa can be observed. While the periodic potential strength is a characteristic of the sample used in the experiment and cannot be varied directly, our finding shows that by varying the bias voltage, change of the periodic potential strength actually experienced by the electrons can be achieved, which can have a huge impact on the experimental investigation of the fractal butterflies in the FQHE region.

The work has been supported by the Canada Research Chairs Program of the Government of Canada.
[†] Electronic address: Tapash.Chakraborty@umanitoba.ca

[2] H. Aoki and M.S. Dresselhaus (Eds.), Physics of Graphene (Springer, New York 2014).

[3] D.S.L. Abergel, V. Apalkov, J. Berashevich, K. Ziegler, and T. Chakraborty, Adv. Phys. 59, 261 (2010).

[4] D. Hofstadter, Phys. Rev. B 14, 2239 (1976), see also, M.Y. Azbel, Sov. Phys. JETP 19, 634 (1964); D. Langbein, Phys. Rev. 180, 633 (1969).

[5] For earlier experimental attempts in semiconductors, see, for example, M.C. Geisler, J.H. Smet, V. Umansky, K.von Klitzing, B. Naundorf, R. Ketzmerick, and H. Schweizer, Phys. Rev. Lett. 92, 256801 (2004); Physica E 25, 227 (2004); C. Albrecht, J.H. Smet, K. von Klitzing, D. Weiss, V.Umansky, and H. Schweitzer, Phys. Rev. Lett. 86, 147 (2001); Physica E 20, 143 (2003); T. Schlösser, K. Ensslin, J.P. Kotthaus, and M. Holland, Europhys. Lett. 33, 683 (1996); Semicond. Sci. Technol. 11, 1582 (1996).
[6] C.R. Dean, L. Wang, P. Maher, C. Forsythe, F. Ghahari, Y. Gao, J. Katoch, M. Ishigami, P. Moon, M. Koshino, T. Taniguchi, K.Watanabe, K.L. Shepard, J.Hone, and P. Kim, Nature 497, 598 (2013).

[7] B. Hunt, J.D. Sanchez-Yamagishi, A.F. Young, M. Yankowitz, B.J. LeRoy, K. Watanabe, T. Taniguchi, P. Moon, M. Koshino, P. Jarillo-Herrero, and R.C. Ashoori, Science 340, 1427 (2013).

[8] L.A. Ponomarenko, R.V. Gorbachev, G.L. Yu, D.C. Elias, R. Jalil, A.A. Patel, A. Mishchenko, A.S. Mayorov, C.R. Woods, J.R. Wallbank, M. Mucha-Kruczynski, B.A. Piot, M. Potemski, I.V. Grigorieva, K.S. Novoselov, F. Guinea, V.I. Falko and A.K. Geim, Nature 497, 594 (2013).

[9] For a brief review on fractal butterflies in monolayer and bilayer graphene, see T. Chakraborty and V.M. Apalkov, IET Circuits Devices Syst., (2015); arXiv:1408.4485 (2014). 
[10] A. Avila and S. Jitomirskaya, Ann. Math. 170, 303 (2009); in Mathematical Physics of Quantum Mechanics (Eds.) Joachim Asch, Alain Joye (Springer, Berlin 2006), Ch. 1, pp. 5-16.

[11] V.M. Apalkov and T. Chakraborty, Phys. Rev. Lett. 112, 176401 (2014).

[12] G.L. Yu, R.V. Gorbachev, J.S. Tu, A.V. Kretinin, Y. Cao, R. Jalil, F. Withers, L.A. Ponomarenko, B.A. Piot, M. Potemski, D.C. Elias, X. Chen, K. Watanabe, T. Taniguchi, I.V. Grigorieva, K.S. Novoselov, V.I. Falko, A.K. Geim, and A. Mishchenko, Nat. Phys. 10, 525 (2014).

[13] T. Chakraborty and V. Apalkov, in [2] Ch. 8; T. Chakraborty and V.M. Apalkov, Solid State Commun. 175, 123 (2013).

[14] V. Apalkov and T. Chakraborty, Solid State Commun. 177, 128 (2014); D.S.L. Abergel and T. Chakraborty, Phys. Rev. Lett. 102, 056807 (2009); D. Abergel, V. Apalkov, and T. Chakraborty, Phys. Rev. B 78, 193405 (2008); D. Abergel, P. Pietiläinen, and T. Chakraborty, Phys. Rev.B 80, 081408 (2009); V. Apalkov and T. Chakraborty, Phys. Rev. B 86, 035401 (2012).

[15] T. Chakraborty and P. Pietiläinen, The Quantum Hall Effects (Springer, New York 1995); T. Chakraborty, and P. Pietiläinen, The Fractional Quantum Hall Effect (Springer, New York 1988); V.M. Apalkov, T. Chakraborty, P. Pietiläinen, Phys. Rev. Lett. 86, 1311 (2001); T. Chakraborty and P. Pietiläinen, ibid. 76, 4018 (1996).

[16] V.M. Apalkov and T. Chakraborty, Phys. Rev. Lett. 97, 126801 (2006).

[17] V.M. Apalkov and T. Chakraborty, Phys. Rev. Lett. 105, 036801 (2010); Phys. Rev. Lett. 107, 186803 (2011).

[18] X. Du, I. Skachko, F. Duerr, A. Luican, and E.Y. Andrei, Nature 462. 192 (2009); D.A. Abanin, I. Skachko, X. Du, E.Y. Andrei, and L.S. Levitov, Phys. Rev. B 81, 115410 (2010); K.I. Bolotin, F. Ghahari, M.D. Shulman, H.L. Störmer, and P. Kim, Nature 462, 196 (2009); F.
Ghahari, Y. Zhao, P. Cadden-Zimansky, K. Bolotin, and P. Kim, Phys. Rev. Lett. 106, 046801 (2011).

[19] A. Ghazaryan, T. Chakraborty, and P. Pietiläinen, arXiv:1408.3424 (2014).

[20] U. Rössler and M. Shurke, in Advances in Solid State Physics, edited by B. Kramer (Springer, Berlin 2000), Vol. 40, pp. 35-50.

[21] V. Gudmundsson and R.R. Gerhardts, Surf. Sci. 361362, 505 (1996); Phys. Rev. B 52, 16744 (1995); Phys. Rev. B 54, 5223R (1996).

[22] M. Koshino and T. Ando, J. Phys. Soc. Jpn 73, 3243 (2004).

[23] E. McCann and V. Falko, Phys. Rev. Lett. 96086805 (2006); E. McCann, Phys. Rev. B 74161403 (2006); E. McCann and M. Koshino, Rep. Prog. Phys. 76056503 (2013).

[24] The periodic rectangular geomery was extensively used earlier in the study of the FQHE in various situations. For example, see T. Chakraborty, Surf. Sci. 229, 16 (1990); Adv. Phys. 49, 959 (2000); T. Chakraborty and P. Pietiläinen, Phys. Rev. Lett. 76, 4018 (1996); T. Chakraborty and P. Pietiläinen, Phys. Rev. Lett. 83, 5559 (1999); T. Chakraborty and P. Pietiläinen, Phys. Rev. B 39, 7971 (1989); V.M. Apalkov, T. Chakraborty, P. Pietiläinen, and K. Niemelä, Phys. Rev. Lett. 86, 1311 (2001); T. Chakraborty, P. Pietiläinen, and F.C. Zhang, Phys. Rev. Lett. 57, 130 (1986); T. Chakraborty and F.C. Zhang, Phys. Rev. B 29, 7032 (R) (1984); F.C. Zhang and T. Chakraborty, Phys. Rev. B 30, 7320 (R) (1984).

[25] A. Ghazaryan and T. Chakraborty, arXiv:1412.2792 (2014).

[26] M. Koshino and E. McCann, Phys. Rev. B 81, 115315 (2010); P. Moon and M. Koshino, Phys. Rev. B 90, 155406 (2014).

[27] F.D.M. Haldane, Phys. Rev. Lett. 55, 2095 (1985).

[28] A. Kol and N. Read, Phys. Rev. B 48, 8890 (1993). 